\title{
High plasma renin activity associates with obesity-related diabetes and arterial hypertension, and predicts persistent hypertension after bariatric surgery
}

\author{
Lucia La Sala ${ }^{{ }^{*}}$, Elena Tagliabue ${ }^{2}$, Elaine Vieira ${ }^{3}$, Antonio E Pontiroli ${ }^{4^{*}}$ (D) and Franco Folli, ${ }^{4,5}$
}

\begin{abstract}
Background: Information about the renin-angiotensin-aldosterone system (RAAS) in obese individuals before and after bariatric surgery is scarce. Aim of this study was to analyze the RAAS in severely obese subjects, in relation to anthropometric and metabolic variables, with special reference to glucose tolerance.

Methods: 239 subjects were evaluated at baseline, and 181 one year after bariatric surgery [laparoscopic gastric banding (LAGB)].

Results: At baseline, renin (plasma renin activity, PRA) was increased from normal to glucose tolerance and more in diabetes, also correlating with ferritin. After LAGB, the decrease of PRA and aldosterone was significant in hypertensive, but not in normotensive subjects, and correlatied with decrease of ferritin. PRA and glucose levels were predictive of persistent hypertension 1 year after LAGB.

Conclusions: These data support the role of RAAS in the pathophysiology of glucose homeostasis, and in the regulation of blood pressure in obesity. Ferritin, as a proxy of subclinical inflammation, could be another factor contributing to the cross-talk between RAAS and glucose metabolism.
\end{abstract}

Keywords: Renin, Aldosterone, Diabetes, HOMA index, Hypertension, Obesity, Weight loss, Bariatric surgery, Laparoscopic gastric banding (LAGB), Ferritin

\section{Introduction}

Arterial hypertension is a major risk factor for all cardiovascular diseases (CVD), including coronary heart disease (CHD), stroke, atrial fibrillation, heart failure (HF), aortic and peripheral arterial disease, and valvular heart disease [1]. Individuals with hypertension have a two- to threefold increased risk for all CVD events combined,

\footnotetext{
*Correspondence: lucia.lasala@multimedica.it; antonio.pontiroli@unimi.it ${ }^{1}$ Lab. of Cardiovascular and Dysmetabolic Disease, IRCCS MultiMedica, 20138 Milan, Italy

${ }^{4}$ Dipartimento di Scienze della Salute, Università degli Studi di Milano, 20142 Milan, Italy

Full list of author information is available at the end of the article
}

as compared with non-hypertensive individuals [2]. The association between obesity and hypertension was shown prospectively in the Framingham Heart Study in the 1960 s, when peripheral resistance was seen in obese hypertensive patients compared with normotensive obese patients [3].

Many factors are implicated in the link of central obesity with pathogenesis of hypertension, such as overactivation of the sympathetic nervous system (SNS) by insulin also present in obese patients [4], leptin, activation of the renin-angiotensin-aldosterone system (RAAS), sodium excretion, pressure natriuresis, and salt sensitivity [5, 6].

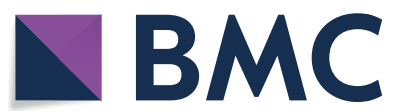

(c) The Author(s) 2021. This article is licensed under a Creative Commons Attribution 4.0 International License, which permits use, sharing, adaptation, distribution and reproduction in any medium or format, as long as you give appropriate credit to the original author(s) and the source, provide a link to the Creative Commons licence, and indicate if changes were made. The images or other third party material in this article are included in the article's Creative Commons licence, unless indicated otherwise in a credit line to the material. If material is not included in the article's Creative Commons licence and your intended use is not permitted by statutory regulation or exceeds the permitted use, you will need to obtain permission directly from the copyright holder. To view a copy of this licence, visit http://creativeco mmons.org/licenses/by/4.0/. The Creative Commons Public Domain Dedication waiver (http://creativecommons.org/publicdomain/ zero/1.0/) applies to the data made available in this article, unless otherwise stated in a credit line to the data. 
As the prevalence of obesity increases, also its comorbidities [arterial hypertension, sleep apnea, impaired glucose tolerance (IGT) type 2 diabetes (T2D) with their associated cardiovascular (CV) risk], will increase as well. These co-morbidities are probably inter-related, due to hyperleptinemia and leptin resistance, oxidative stress, sub-clinical inflammation, endothelial dysfunction, sympathetic activation, insulin resistance (IR), and overactivation of RAAS [7]. In contrast, there are no data on the interplay between glucose tolerance and RAAS in obesity, in spite of the known cross-talk between insulin resistance and RAAS $[8,9]$.

Weight loss, obtained with diet alone, or in combination with pharmacological treatment, or bariatric surgery (BS), improves several metabolic variables and reduces arterial blood pressure $[10,11]$, preventing the appearance of T2D [12, 13] and of arterial hypertension [10]; in addition, BS, compared with medical treatment, improves quality of life and life expectancy $[14,15]$ and is cost-effective in the management of obesity $[16,17]$. Most abnormalities improve after weight loss, and the evidences that these changes are related to each other and mainly with loss of abdominal fat are known [18]. Although previous data supported the benefits of laparoscopic gastric banding (LAGB) in greatly reducing risk factors for cardiovascular disease [19], the mechanisms through which weight reduction decreases the cardiovascular risk are not well established. There are some reports showing that weight loss, obtained through BS, reduces blood pressure, aldosterone (ALD) and renin plasma levels in hypertensive subjects, not in normotensive subjects [20]. It is well known that ALD affects glucose metabolism homeostasis and its high levels may increase the risk for diabetes mellitus (MESA, Multiethnic Study of Atherosclerosis) [21]. The pharmacological modulation of RAAS might be helpful in lowering the risk of appearance of diabetes and of hypertension [22, 23].

The first aim of this study was to analyze RAAS in obese subjects, candidate for BS, and to correlate RAAS with anthropometric and metabolic variables, especially with glucose tolerance. The second aim of this study was to analyze changes of basal components of RAAS system, of glucose metabolism, and of insulin sensitivity in obese subjects after bariatric surgery; the third aim was to develop predictive models of persistence of diabetes and of hypertension in spite of weight loss.

\section{Subjects and methods}

239 obese subjects (43 men and 196 women, aged $40.6 \pm 10.4$ years, baseline BMI $44.5 \pm 6.4 \mathrm{~kg} / \mathrm{m}^{2}$ ), candidates for LAGB, were included in the study; all subjects gave written informed consent following the common protocol already described [24] approved by the local
Ethics Committee, and were studied as in-patients under standardized conditions. All evaluations were performed at 30-45 days before intervention. Height, weight and waist circumference were measured in all subjects. Except for 6 patients with a pre-existing diagnosis of diabetes, diagnosis of was done by oral glucose tolerance test (OGTT) on fasting and/or 2-h plasma glucose [24]. Blood pressure was measured with an appropriate cuff three times in resting conditions in supine position, and the average of the second two readings was recorded. Hypertension was defined as systolic blood pressure $(\mathrm{SBP})>140 \mathrm{mmHg}$ and/or diastolic pressure $(\mathrm{DBP})>90$ $\mathrm{mmHg}$ [25] or current treatment with anti-hypertensive drugs (three patients). Supine (after $2 \mathrm{~h}$ in lying position) and upright ( $2 \mathrm{~h}$ after standing) plasma renin activity (PRA) and ALD, fasting plasma glucose (FPG), insulin, HbA1c, lipid profile, sodium and potassium electrolytes, ferritin, and creatinine were evaluated at $8.00 \mathrm{am}$ under fasting conditions. The HOMA index (HOMA-IR) was also calculated [26]. Secondary hypertension was excluded on the basis of clinical and instrumental diagnosis; in particular, subjects with an elevated upright ALD/PRA ratio [27] underwent further investigation to rule out primary hyperaldosteronism, and were excluded from the study. Urinary cortisol levels were also assayed $[28,29]$. All subjects underwent LAGB, and 181 of them could be analyzed 12 months after surgery. The post-surgery diet has already been described [24].

\section{Biochemical and hormonal assays}

PRA and ALD were measured by radioimmunoassay (RIA) methods (DiaSorin, Vercelli, Italy, and MedicalSystem SpA, Genova, Italy, respectively). Serum electrolytes, blood glucose, insulin, creatinine, HbA1c, total and HDL cholesterol, triglycerides, leptin, ferritin, iron, and transferrin were evaluated as already indicated [24, 30, 31]. Urinary free cortisol levels were measured by immunologic chemiluminescent assay (Advia Centaur Bayer, BAYER- Diagnostics). All measurements were carried out in the same laboratory; intra- and inter-assay coefficients of variation (CVs) for insulin, PRA, and ALD assay were $3.0-5.0 \%, 3.6-6.1 \%$, and $3.3-5.6 \%$, respectively.

\section{Statistical analysis}

Data are presented as mean \pm SD or as frequencies. Subjects were divided into normal glucose tolerance (NGT), impaired glucose tolerance (IGT) and T2D. Intrasubject changes between baseline and after 12 months from LAGB were analyzed by paired $t$ test or non-parametric Wilcoxon signed rank test, as appropriate. Since a normal distribution of data was not assured, data were compared in the glucose tolerance groups by non-parametric Kruskal-Wallis test followed by Bonferroni adjustment 
for multiple comparisons. Normotensive and hypertensive were compared by non-parametric Wilcoxon test. Correlation between baseline PRA levels and other collected variables was evaluated. Multivariable regression analysis was performed for some outcomes, with models including significant variables at univariate analysis.

\section{Modeling}

Logistic modeling for the persistence of diabetes after LAGB was calculated by taking into account variables significantly associated with diabetes at 1 year. Logistic regression models for the association between hypertension at 12 months after LAGB and PRA, adjusted for glycemic parameters or groups were implemented and correspondent receiver operating characteristic (ROC) curves, with AUC (area under the ROC curve), were drawn. Based on the logistic model, a nomogram was built to predict the probability to have persistence of arterial hypertension at 1 year after BS, in spite of weight loss. $p$ less than 0.05 were considered statistically significant; for multiple comparisons, a $p$ value $<0.0167$ was considered statistically significant. All statistical analyses were performed with SAS Software v 9.4 (SAS Institute Inc., Cary, NC, USA) or STATA12 for Macintosh (Stata Corporation, College Station, TX).

\section{Results}

\section{Baseline conditions}

At baseline NGT subjects were younger than IGT and T2DM subjects, without differences in sex proportion and BMI among groups. Triglycerides were different among the three groups; in addition, diastolic BP, ferritin were higher for T2D than IGT and NGT, and HDLcholesterol were lower; the frequency of hypertensive subjects was higher for IGT and T2D than NGT. Transferrin, iron, and albumin did not differ among the three groups. There was a trend towards increased supine PRA between NGT, IGT and T2D, although not significant (Table 1).

Table 2 shows that hypertensive subjects $(\mathrm{n}=70)$ were older, with higher BP and supine PRA than normotensive subjects $(n=169)$. In addition, for waist circumference, total cholesterol, triglycerides, ferritin, uric acid and glycemic parameters except that insulin, differences were significant between normotensive and hypertensive subjects. Baseline supine PRA levels were significantly correlated with age, glycemic parameters, leptin, ferritin, calcium, sodium, ALD and uric acid (Table 3). Reverse associations were also calculated; HOMA-IR, FPG and $1 \mathrm{hPPg}$ and 2hPPG correlated with ferritin. At multivariate analysis, glucose tolerance, blood glucose, HOMA-IR, and ferritin were predictors of supine and upright PRA; age and supine PRA were predictors of supine ALD, and age, upright PRA and blood glucose were predictors of upright ALD (results not shown).

\section{Effects of surgery}

In subjects re-evaluated one year after BS, all changes observed were as expected. Subjects re-evaluated after one year differed from subjects lost to follow-up only for age ( $41.96 \pm 10.13$ vs. $36.30 \pm 9.96$ year, $\mathrm{p}=0.0002)$, not for any other variable (data not shown). Weight, BMI, waist circumference, glycemic parameters, triglycerides and BP significantly decreased after LAGB (Table 4). Also supine PRA (Table 4; Fig. 1), supine ALD and ferritin significantly decreased after bariatric BS. Particularly, weight, BMI, waist circumference and glycemic parameters decreased in a similar manner in NGT, IGT, and T2DM subjects (Table 5). Triglycerides, HDL and BP significantly improve after LAGB in all groups, except for SBP and DBP that did not change in IGT subjects. Generally, NGT was the group in which changes after BS were most evident, including hypertension. Metabolic and glycemic parameters significantly decreased after LAGB in both normotensive and hypertensive subjects (Table 6). Also HDL, triglycerides, BP and ferritin improved after intervention, but transferrin, iron and albumin did not vary. Particularly, hypertensive subjects were those with the greatest changes. Supine PRA and ALD only significantly decreased in hypertensive subjects, not in normotensive subjects. Both glucose tolerance and frequency of hypertension improved after surgery, but a few patients required de-novo treatment of hypertension. At multivariable analysis, decrease of supine PRA and of upright PRA was predicted by decrease of ferritin; decrease of both supine and upright ALD was predicted by decrease of waist circumference (data not shown).

\section{Modelling}

The only predictor of diabetes 1 year after BS was represented by fasting blood glucose (or 1hPPG or 2hPPG) or glucose tolerance at baseline. Of the patients with hypertension at 1 year after LAGB, 29 were hypertensive at baseline and 2 became hypertensive during the follow-up. Association between hypertension at 12 months from LAGB and baseline supine PRA was evaluated by three logistic regression models in which supine PRA was adjusted either by glucose tolerance groups, FPG and 2hPG (Model 1, Model 2 and Model 3, respectively-Table 7). Increase in supine PRA levels was associated with probability of hypertension at 12 months, which ranged from 47 to $63 \%$, for each $\mathrm{ng} / \mathrm{ml} / \mathrm{h}$ of PRA increase. In Model 1, IGT subjects had 3.60 times probability to be hypertensive at 12 months after surgery as compared with NGT. That probability increased for T2D if compared with NGT (OR: 4.85; 95\% CI 1.15-20.56). 
Table 1 Baseline details of all subjects in the study divided by glucose tolerance

\begin{tabular}{|c|c|c|c|c|c|c|c|}
\hline & NGT & IGT & T2D & Overall $p^{\wedge}$ & NGT vs. IGT* & NGT vs. T2D* & IGT vs. T2D* \\
\hline$N(M / W)$ & $139(26 / 113)$ & $66(10 / 56)$ & $34(7 / 27)$ & 0.754 & & & \\
\hline Age (yr) & $37.5 \pm 9.6$ & $43.2 \pm 10.0$ & $46.2 \pm 8.3$ & $<0.0001$ & 0.0002 & $<0.0001$ & 0.2313 \\
\hline Weight (kg) & $118.4 \pm 20.3$ & $119.4 \pm 20.3$ & $123.0 \pm 21.4$ & 0.4000 & 0.7711 & 0.1883 & 0.2709 \\
\hline $\mathrm{BMI}\left(\mathrm{kg} / \mathrm{m}^{2}\right)$ & $44.0 \pm 5.8$ & $44.2 \pm 5.7$ & $45.6 \pm 5.7$ & 0.1331 & 0.1847 & 0.0746 & 0.4498 \\
\hline Waist circum (cm) & $120.6 \pm 14.3$ & $121.8 \pm 12.4$ & $129.5 \pm 12.9$ & 0.0029 & 0.3278 & 0.0011 & 0.0061 \\
\hline FPG (mg/dl) & $97.3 \pm 12.8$ & $107.6 \pm 18.1$ & $165.5 \pm 70.6$ & $<0.0001$ & 0.0004 & $<0.0001$ & $<0.0001$ \\
\hline $1 \mathrm{hPG}(\mathrm{mg} / \mathrm{dl})$ & $155.4 \pm 44.2$ & $201.0 \pm 47.8$ & $306.8 \pm 94.7$ & $<0.0001$ & $<0.0001$ & $<0.0001$ & $<0.0001$ \\
\hline $2 \mathrm{hPG}(\mathrm{mg} / \mathrm{dl})$ & $107.7 \pm 20.9$ & $163.4 \pm 16.1$ & $286.0 \pm 82.1$ & $<0.0001$ & $<0.0001$ & $<0.0001$ & $<0.0001$ \\
\hline Insulin $(\mu \mathrm{U} / \mathrm{ml})$ & $18.9 \pm 11.8$ & $18.1 \pm 9.0$ & $23.5 \pm 16.9$ & 0.1374 & 0.8736 & 0.0542 & 0.0844 \\
\hline HOMA-IR & $4.6 \pm 3.4$ & $4.9 \pm 2.9$ & $9.2 \pm 6.2$ & $<0.0001$ & 0.2888 & $<0.0001$ & $<0.0001$ \\
\hline HbA1c (\%) & $5.8 \pm 0.6$ & $6.2 \pm 0.7$ & $7.8 \pm 1.8$ & $<0.0001$ & 0.0006 & $<0.0001$ & $<0.0001$ \\
\hline Total cholesterol (mg/dl) & $201.3 \pm 40.2$ & $206.8 \pm 40.2$ & $215.1 \pm 50.0$ & 0.2396 & 0.2995 & 0.1225 & 0.4683 \\
\hline HDL-cholesterol (mg/dl) & $48.5 \pm 12.0$ & $50.6 \pm 18.0$ & $41.8 \pm 10.6$ & 0.0126 & 0.9916 & 0.0051 & 0.0067 \\
\hline Triglycerides (mg/dl) & $131.5 \pm 72.1$ & $144.9 \pm 67.5$ & $219.3 \pm 115.6$ & $<0.0001$ & 0.0418 & $<0.0001$ & 0.0010 \\
\hline Systolic BP (mmHg) & $131.5 \pm 15.4$ & $131.4 \pm 13.3$ & $138.1 \pm 17.6$ & 0.1066 & 0.8417 & 0.0395 & 0.0761 \\
\hline Diastolic BP (mmHg) & $82.4 \pm 8.4$ & $82.8 \pm 9.4$ & $87.8 \pm 10.9$ & 0.0113 & 0.8700 & 0.0032 & 0.0140 \\
\hline Hypertension (Y/N) & $29 / 110$ & $24 / 42$ & $17 / 17$ & 0.0019 & 0.0258 & 0.0011 & 0.4431 \\
\hline Creatinine (mg/dl) & $0.7 \pm 0.1$ & $0.7 \pm 0.1$ & $0.7 \pm 0.2$ & 0.4839 & 0.2285 & 0.9787 & 0.4878 \\
\hline Leptin (ng/ml) & $37.7 \pm 18.4$ & $41.3 \pm 18.2$ & $43.2 \pm 37.1$ & 0.6192 & 0.3873 & 0.7705 & 0.5264 \\
\hline Ferritin (ng/ml) & $86.1 \pm 92.1$ & $83.6 \pm 93.6$ & $154.3 \pm 178.2$ & 0.0919 & 0.8618 & 0.0435 & 0.0362 \\
\hline Transferrin (mg/ml) & $2.7 \pm 0.45$ & $2.7 \pm 0.53$ & $2.8 \pm 0.58$ & 0.8545 & 0.7821 & 0.5779 & 0.7808 \\
\hline Iron ( $\mu \mathrm{g} / \mathrm{dl})$ & $84.2 \pm 33.15$ & $78.9 \pm 28.59$ & $81.4 \pm 26.65$ & 0.5331 & 0.8963 & 0.3110 & 0.5068 \\
\hline Albumin (g/l) & $58.2 \pm 4.44$ & $58.0 \pm 3.63$ & $56.6 \pm 5.44$ & 0.1826 & 0.8187 & 0.0855 & 0.1274 \\
\hline Calcium (mmol/l) & $2.3 \pm 0.1$ & $2.3 \pm 0.1$ & $2.3 \pm 0.1$ & 0.2797 & 0.1082 & 0.6496 & 0.4999 \\
\hline Sodium (mmol/l) & $140.6 \pm 2.3$ & $140.6 \pm 2.3$ & $140.3 \pm 2.9$ & 0.7137 & 0.8625 & 0.4656 & 0.4203 \\
\hline Supine PRA (ng/ml/h) & $1.8 \pm 2.3$ & $2.2 \pm 4.2$ & $4.3 \pm 8.0$ & 0.2543 & 0.2775 & 0.3262 & 0.1229 \\
\hline Upright PRA (ng/ml/h) & $3.2 \pm 3.9$ & $3.5 \pm 5.2$ & $7.5 \pm 13.5$ & 0.4492 & 0.2339 & 0.9617 & 0.3440 \\
\hline Supine ALD (ng/dl) & $3.6 \pm 2.7$ & $3.1 \pm 2.7$ & $3.4 \pm 1.9$ & 0.1871 & 0.0932 & 0.8555 & 0.1464 \\
\hline Upright ALD (ng/dl) & $8.4 \pm 4.7$ & $7.2 \pm 5.0$ & $9.7 \pm 7.4$ & 0.0739 & 0.0289 & 0.8127 & 0.1123 \\
\hline Upright ALD/PRA & $5.0 \pm 6.5$ & $5.8 \pm 9.2$ & $5.2 \pm 7.0$ & 0.9172 & 0.9399 & 0.6749 & 0.7665 \\
\hline Urinary cortisol (ng/24 h) & $117.6 \pm 46.9$ & $115.2 \pm 42.1$ & $110.2 \pm 51.4$ & 0.5540 & 0.7934 & 0.2833 & 0.4302 \\
\hline Uric acid (mg/dl) & $5.1 \pm 1.2$ & $5.0 \pm 1.1$ & $5.4 \pm 1.4$ & 0.5677 & 0.5804 & 0.4695 & 0.2901 \\
\hline Hypoglycemic therapy ${ }^{a}$ & $0.0 \pm 0.0$ & $0.0 \pm 0.0$ & $1.5 \pm 3.3$ & $<0.0001$ & 1.0000 & $<0.0001$ & $<0.0001$ \\
\hline
\end{tabular}

NGT normal glucose tolerance, IGT impaired glucose tolerance, T2D type 2 diabetes, BMI body mass index, FPG fasting plasma glucose, 1 hPG 1 -hour plasma glucose, 2hPG 2-hour plasma glucose, BP blood pressure, $P R A$ plasma renin activity, $A L D$ aldosterone

${ }^{\wedge}$ Non-parametric Kruskal-Wallis test

*Multiple comparisons are based on pairwise Wilcoxon test with Bonferroni adjustment $(p<0.0167$ is considered statistically significant)

a Pills per day

Model 2 and 3 showed that for each $\mathrm{mg} / \mathrm{dl}$ increase of FPG or 2hPG, the probability of hypertension at 12 months after LAGB increased of 2 and 1\%, respectively. Figure 2 shows ROC curves and AUC for each logistic model. The nomogram based on Model 2 is a useful tool to predicted probability hypertension at 12 months from BS basing on subject characteristics. For instance, a subject with supine PRA $=5.0 \mathrm{ng} / \mathrm{ml} / \mathrm{h}$ and $\mathrm{FPG}=250 \mathrm{mg} /$ $\mathrm{dl}$ has about $90 \%$ risk of hypertension at 1 year, despite weight loss (Fig. 3).

\section{Discussion}

In this study, in obese subjects undergoing bariatric surgery, supine PRA was higher in hypertensive than in normotensive subjects, and progressively higher in normal glucose tolerance, impaired glucose tolerance, and diabetes. Before BS, supine PRA correlated with HOMA-IR, and both PRA and HOMA-IR correlated with ferritin. Interestingly, decrease of supine PRA at 12 months after LAGB only occurred in hypertensive subjects, as previously reported, with no difference among groups 
Table 2 Baseline details of all subjects in the study divided by hypertension

\begin{tabular}{|c|c|c|c|}
\hline & Normotensive & Hypertensive & $p^{*}$ \\
\hline$N(M / W)$ & 169 & 70 & \\
\hline Age (yr) & $38.2 \pm 9.7$ & $46.4 \pm 9.7$ & $<0.0001$ \\
\hline Weight (kg) & $117.7 \pm 19.2$ & $122.7 \pm 21.7$ & 0.1454 \\
\hline $\mathrm{BMI}\left(\mathrm{kg} / \mathrm{m}^{2}\right)$ & $44.0 \pm 6.2$ & $45.8 \pm 6.8$ & 0.0687 \\
\hline Waist circumference $(\mathrm{cm})$ & $120.3 \pm 13.5$ & $127.0 \pm 13.7$ & 0.0005 \\
\hline FPG (mg/dl) & $103.1 \pm 23.9$ & $126.6 \pm 55.8$ & $<0.0001$ \\
\hline $1 \mathrm{hPG}(\mathrm{mg} / \mathrm{dl})$ & $175.1 \pm 55.8$ & $222.4 \pm 99.7$ & 0.0006 \\
\hline $2 \mathrm{hPG}(\mathrm{mg} / \mathrm{dl})$ & $136.3 \pm 52.8$ & $177.6 \pm 93.4$ & 0.0004 \\
\hline Insulin $(\mu \mathrm{U} / \mathrm{ml})$ & $19.3 \pm 13.2$ & $19.0 \pm 9.0$ & 0.3967 \\
\hline HOMA-IR & $5.0 \pm 4.0$ & $6.1 \pm 4.3$ & 0.0240 \\
\hline HbA1c (\%) & $6.0 \pm 0.9$ & $6.7 \pm 1.4$ & $<0.0001$ \\
\hline $\begin{array}{l}\text { Glucose tolerance (NGT/ } \\
\text { IGT/T2D) }\end{array}$ & $110 / 42 / 17$ & 29/24/17 & 0.0019 \\
\hline Total cholesterol (mg/dl) & $199 \pm 40.5$ & $215.5 \pm 42.1$ & 0.0025 \\
\hline HDL-cholesterol (mg/dl) & $48.3 \pm 14.3$ & $47.6 \pm 13.2$ & 0.7593 \\
\hline Triglycerides (mg/dl) & $137.7 \pm 72.2$ & $174.0 \pm 100.5$ & 0.0046 \\
\hline Systolic BP (mmHg) & $126.2 \pm 9.0$ & $143.3 \pm 17.9$ & $<0.0001$ \\
\hline Diastolic BP (mmHg) & $79.7 \pm 6.6$ & $89.5 \pm 10.0$ & $<0.0001$ \\
\hline Creatinine (mg/dl) & $0.7 \pm 0.1$ & $0.7 \pm 0.2$ & 0.1204 \\
\hline Leptin (ng/ml) & $40.1 \pm 19.4$ & $38.6 \pm 21.9$ & 0.4670 \\
\hline Ferritin (ng/ml) & $76.5 \pm 83.6$ & $135.6 \pm 140.6$ & 0.0004 \\
\hline Transferrin (mg/ml) & $2.8 \pm 0.5$ & $2.6 \pm 0.5$ & 0.0637 \\
\hline Iron ( $\mu \mathrm{g} / \mathrm{dl})$ & $82.1 \pm 33.7$ & $82.8 \pm 25.3$ & 0.8775 \\
\hline Albumin (g/l) & $57.7 \pm 4.23$ & $58.3 \pm 4.7$ & 0.3213 \\
\hline Calcium (mmol/l) & $2.3 \pm 0.1$ & $2.4 \pm 0.1$ & 0.1770 \\
\hline Sodium (mmol/l) & $140.6 \pm 2.5$ & $140.3 \pm 2.3$ & 0.5040 \\
\hline Supine PRA (ng/ml/h) & $1.7 \pm 3.0$ & $4.1 \pm 6.9$ & 0.0159 \\
\hline Upright PRA (ng/ml/h) & $3.0 \pm 4.1$ & $6.9 \pm 10.9$ & 0.1200 \\
\hline Supine ALD (ng/dl) & $3.3 \pm 2.2$ & $3.5 \pm 3.1$ & 0.6808 \\
\hline Upright ALD (ng/dl) & $7.9 \pm 4.5$ & $9.0 \pm 6.5$ & 0.4968 \\
\hline Upright ALD/PRA & $4.8 \pm 5.1$ & $5.9 \pm 10.6$ & 0.0644 \\
\hline Urinary cortisol (ng/24 h) & $113.8 \pm 45.8$ & $115.1 \pm 47.3$ & 0.8875 \\
\hline Uric acid (mg/dl) & $5.0 \pm 1.2$ & $5.5 \pm 1.2$ & 0.0022 \\
\hline Hypoglycemic therapy ${ }^{a}$ & $0.2 \pm 1.4$ & $0.4 \pm 1.4$ & 0.0176 \\
\hline
\end{tabular}

$B M /$ body mass index, $F P G$ fasting plasma glucose, 1 hPG 1-hour plasma glucose, 2hPG 2-hour plasma glucose, NGT normal glucose tolerance, IGT impaired glucose tolerance, $T 2 D$ type 2 diabetes, BP blood pressure, PRA plasma renin activity, $A L D$ aldosterone

*Non-parametric Wilcoxon test

${ }^{a}$ Pills per day

of glucose tolerance. Also, decrease of supine PRA correlated with decrease of HOMA-IR and with decrease of ferritin, suggesting that changes of ferritin are pivotal to change of HOMA-IR and of supine PRA. Similar, albeit less significant, was the behavior of upright PRA, and of both supine and upright aldosterone. Obese individuals frequently develop hypertension, which is largely attributable to RAAS overactivity [6]. Logistic modeling
Table 3 Correlations between baseline characteristics and supine renin activity

\begin{tabular}{|c|c|c|c|}
\hline & $\mathrm{N}$ & Rho & $p$ \\
\hline Age (yr) & 225 & 0.14707 & 0.0274 \\
\hline $\mathrm{FPG}(\mathrm{mg} / \mathrm{dl})$ & 219 & 0.39719 & $<0.0001$ \\
\hline $1 \mathrm{hPG}(\mathrm{mg} / \mathrm{dl})$ & 217 & 0.32632 & $<0.0001$ \\
\hline $2 \mathrm{hPG}(\mathrm{mg} / \mathrm{dl})$ & 216 & 0.29338 & $<0.0001$ \\
\hline HOMA-IR & 217 & 0.20082 & 0.0032 \\
\hline HbA1c (\%) & 217 & 0.37418 & $<0.0001$ \\
\hline Leptin (ng/ml) & 50 & 0.29842 & 0.0353 \\
\hline Ferritin (ng/ml) & 218 & 0.25217 & 0.0004 \\
\hline Calcium (mmol/L) & 192 & 0.21115 & 0.0033 \\
\hline Sodium (mmol/l) & 225 & -0.21633 & 0.0011 \\
\hline Upright PRA (ng/ml/h) & 224 & 0.89527 & $<0.0001$ \\
\hline Supine ALD (ng/dl) & 216 & 0.23005 & 0.0007 \\
\hline Upright ALD (ng/dl) & 216 & 0.26412 & $<0.0001$ \\
\hline Upright ALD/PRA & 215 & -0.23321 & 0.0006 \\
\hline Uric acid (mg/dl) & 218 & 0.17363 & 0.0102 \\
\hline Hypoglycemic therapy ${ }^{a}$ & 190 & 0.19768 & 0.0063 \\
\hline
\end{tabular}

Only significant correlations are shown

FPG fasting plasma glucose, 1 hPG 1-hour plasma glucose, 2hPG 2-hour plasma glucose, $P R A$ plasma renin activity, $A L D$ aldosterone

${ }^{a}$ Pills per day

demonstrated that glucose tolerance (or FPG or 2hPPG) and PRA are predictors of persistent hypertension at 1 year after BS; a nomogram was derived with probabilities of persistent hypertension based on FPG and PRA at baseline. These results also point to a contribution of ferritin in the interplay between the RAAS and insulin sensitivity. However, there are other aspects that deserve consideration in this possible interplay. For instance, BS is also accompanied by reduced sub-clinical inflammation (not evaluated in this study); studies have shown decrease of inflammatory markers after BS [32], and ferritin is considered also a marker of inflammation [33]. In this study, changes in ferritin levels among NGT, IGT, and DM were not accompanied by changes in transferrin, iron, and albumin. We should not forget the possible role of the hypothalamic-pituitary-adrenal axis in obese individuals [34-36]; in this study, free urinary cortisol was also decreased after BS. Our results are consistent with the demonstrated existence of active cross-talks between angiotensin II and insulin signaling, as well as a role of sub inflammation in contributing to insulin resistance in obese hypertensive subjects [37-41]. Further studies are required for a full comprehension of the interplay between RAAS and insulin sensitivity in obese individuals. The possible role of PRA in the characterization of metabolic phenotype of "remitted obese" is supported by investigations on mice lacking renin $(\operatorname{Ren} 1 c)$, a strain 
Table 4 Differences between baseline values and 12 months after bariatric surgery

\begin{tabular}{|c|c|c|c|c|c|}
\hline & \multicolumn{2}{|c|}{ Baseline } & \multicolumn{2}{|c|}{12 months } & \multirow[t]{2}{*}{$p^{\wedge}$} \\
\hline & $\mathbf{N}$ & Mean $( \pm \mathrm{SD})$ & $\mathrm{N}$ & Mean $( \pm \mathrm{SD})$ & \\
\hline Weight (kg) & 239 & $119.2 \pm 19.97$ & 181 & $97.3 \pm 16.61$ & $<0.0001$ \\
\hline $\mathrm{BMI}\left(\mathrm{kg} / \mathrm{m}^{2}\right)$ & 239 & $44.4 \pm 5.79$ & 181 & $36.4 \pm 5.21$ & $<0.0001$ \\
\hline $\begin{array}{l}\text { Waist circumfer- } \\
\text { ence }(\mathrm{cm})\end{array}$ & 238 & & 174 & & $<0.0001$ \\
\hline FPG (mg/dl) & 233 & $110.4 \pm 38.05$ & 171 & $95.8 \pm 17.33$ & $<0.0001$ \\
\hline $1 \mathrm{hPG}(\mathrm{mg} / \mathrm{dl})$ & 226 & $189.8 \pm 75.31$ & 176 & $158.2 \pm 58.57$ & $<0.0001$ \\
\hline $2 \mathrm{hPG}(\mathrm{mg} / \mathrm{dl})$ & 225 & $149.1 \pm 70.52$ & 169 & $118.7 \pm 47.94$ & $<0.0001^{*}$ \\
\hline Insulin ( $\mu \mathrm{U} / \mathrm{ml})$ & 230 & $19.4 \pm 11.25$ & 160 & $10.0 \pm 5.21$ & $<0.0001$ \\
\hline HOMA-IR & 230 & $5.4 \pm 4.08$ & 147 & $2.4 \pm 1.5$ & $<0.0001$ \\
\hline HbA1c (\%) & 229 & $6.2 \pm 1.1$ & 155 & $5.7 \pm 0.7$ & $<0.0001$ \\
\hline $\begin{array}{l}\text { Total cholesterol } \\
(\mathrm{mg} / \mathrm{dl})\end{array}$ & 239 & $203.9 \pm 41.51$ & 181 & $.7 \pm 42.54$ & $0.4949^{*}$ \\
\hline $\begin{array}{l}\text { HDL-cholesterol } \\
(\mathrm{mg} / \mathrm{dl})\end{array}$ & 225 & $48.1 \pm 1$ & 168 & $53.4 \pm 1$ & $001^{*}$ \\
\hline $\begin{array}{l}\text { Triglycerides (mg/ } \\
\text { dl) }\end{array}$ & 239 & 08 & 171 & $107.4 \pm 5$ & $<0.0$ \\
\hline $\begin{array}{r}\text { Systolic BP } \\
(\mathrm{mmHg})\end{array}$ & 4 & $132.3 \pm 1$ & 136 & $127.1 \pm 12.9$ & (0 \\
\hline $\begin{array}{l}\text { Diastoli } \\
(\mathrm{mml}\end{array}$ & 4 & $83.2 \pm 9.24$ & 136 & $80.3 \pm 8.89$ & 0.0004 \\
\hline Creatinine (mg/dl) & 239 & $0.7 \pm 0.15$ & 167 & $0.7 \pm 0.07$ & 0.2882 \\
\hline Leptin (ng/ml) & 52 & $39.5 \pm 20.22$ & 52 & $20.6 \pm 12.4$ & $<0.0001^{*}$ \\
\hline Ferritin (ng/ml) & 232 & $95.5 \pm 100.98$ & B 181 & $83.3 \pm 84.81$ & 0.0164 \\
\hline $\begin{array}{l}\text { Transferrin (mg/ } \\
\text { ml) }\end{array}$ & 221 & $2.7 \pm 0.49$ & 155 & $26+048$ & 0.3279 \\
\hline Iron ( $\mu \mathrm{g} / \mathrm{dl})$ & 235 & $82.3 \pm 31.08$ & 181 & $85.8 \pm 30.43$ & 0.2195 \\
\hline Albumin (g/l) & 232 & $57.9 \pm 4.40$ & 159 & $58.6 \pm 4.38$ & 0.8788 \\
\hline Calcium (mmol/l) & 202 & $2.3 \pm 0.11$ & 163 & $2.4 \pm 0.11$ & $0.0055^{*}$ \\
\hline Sodium (mmol/l) & 239 & $140.5 \pm 2.42$ & 181 & $141.0 \pm 2.44$ & $0.0014^{*}$ \\
\hline $\begin{array}{l}\text { Supine PRA (ng/ } \\
\mathrm{ml} / \mathrm{h} \text { ) }\end{array}$ & 225 & $2.4 \pm 4.63$ & 115 & $1.3 \pm 2.50$ & 0.0035 \\
\hline $\begin{array}{l}\text { Upright PRA (ng/ } \\
\mathrm{ml} / \mathrm{h} \text { ) }\end{array}$ & 224 & $1 \pm 7.08$ & 116 & $3.1 \pm 6.09$ & 0.1821 \\
\hline $\begin{array}{l}\text { Supine ALD (ng/ } \\
\text { dl) }\end{array}$ & 230 & $3 \pm 2.53$ & 113 & $2.9 \pm 3.1$ & 0.0005 \\
\hline $\begin{array}{l}\text { Upright ALD (ng/ } \\
\text { dl) }\end{array}$ & 230 & $2 \pm 5.25$ & 114 & $3 \pm 8.2$ & 0.0439 \\
\hline Upright ALD/PRA & 215 & $5.2 \pm 7.23$ & 111 & $5.7 \pm 6.9$ & 0.8917 \\
\hline $\begin{array}{l}\text { Urinary cortisol } \\
\text { (ng/24 h) }\end{array}$ & 180 & $114.1 \pm 46.06$ & 96 & $106.8 \pm 45.5$ & $0.0170^{*}$ \\
\hline Uric acid (mg/dl) & 232 & $5.2 \pm 1.20$ & 181 & $4.7 \pm 1.27$ & $<0.0001^{*}$ \\
\hline $\begin{array}{l}\text { Hypoglycemic } \\
\text { therapy }^{\text {a }}\end{array}$ & 239 & $0.2 \pm 1.4$ & 157 & $0.3 \pm 2.2$ & 1.0000 \\
\hline
\end{tabular}

$B M /$ body mass index, $F P G$ fasting plasma glucose, 1 hPG 1-hour plasma glucose, 2hPG 2-hour plasma glucose, $B P$ blood pressure, $P R A$ plasma renin activity, $A L D$ aldosterone

${ }^{\wedge}$ Non-parametric Wilcoxon Signed Rank test for paired data

*Paired T test

a Pills per day

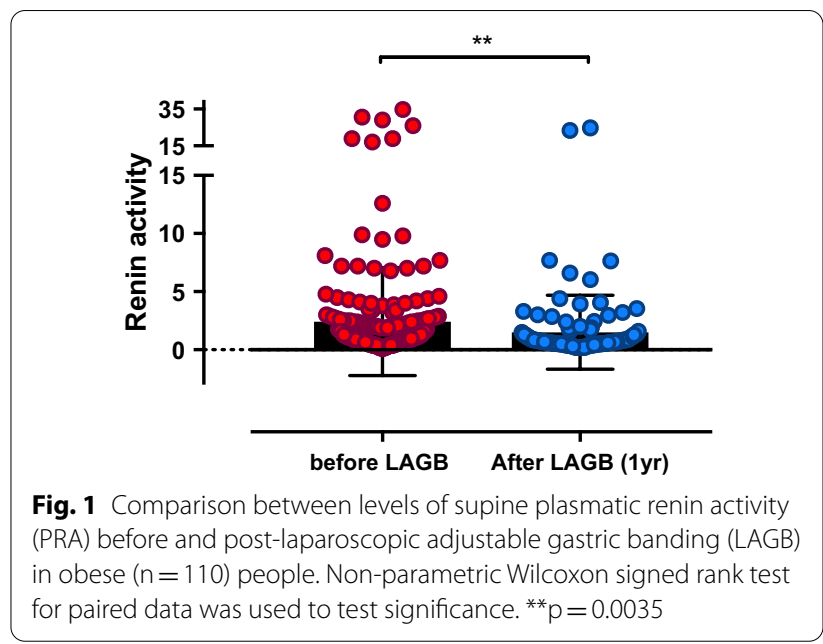

prone to diet-induced obesity. The $R e n 1 c^{-/-}$mice are lean, insulin sensitive, and are resistant to diet-induced obesity without changes in food intake and physical activity. In $\operatorname{Ren} 1 c^{-1-}$ mice enhanced energy expenditure and are resistant to development of HFD-induced obesity [42]. Instead, transgenic rodents overexpressing human renin are obese due to increased food intake and exhibit hyperglycemia, hyperinsulinemia, hyperlipidemia, and insulin resistance [43]. In contrast, the role of gender seems to be related to renin activity; female mice overexpressing human renin are protected from HFDinduced obesity. Furthermore, dysfunctional adipose tissue can partially account for the alterations of RAAS signaling influencing the surrounding organs, including the vasculature. It is well known the paracrine communication between adipose tissue and skeletal muscle, pancreas and cardiovascular system with the release of adipokines, cytokines and other small molecules, such as extracellular vesicles (EV) deputed to vehicle small RNAs (microRNAs) among others [44]. For example, miR-27a, which plays a critical for obesity by regulating insulin resistance in adipocytes, seems to facilitate the crosstalk between adipocytes and skeletal muscle, inducing insulin resistance by PPARG [45]. Bariatric surgery leads to a remission/resolution of $\mathrm{T} 2 \mathrm{D}$, but its precise mechanisms are not yet fully understood and the mechanisms of actions are complex, but it seems that the induction of satiety occurs by direct pressure or contact of the band with the gastric wall, or via vagal nerve signaling [46]. Beside the physiology, it is also true that a small number of studies demonstrated the role of microRNA after BS in providing a specific metabolic pattern [47-49]: 
Table 5 Changes induced by weight loss according to glucose tolerance

\begin{tabular}{|c|c|c|c|c|c|c|c|c|}
\hline & All & $p^{\wedge}$ & NGT & $p^{\wedge}$ & IGT & $p^{\wedge}$ & T2DM & $p^{\wedge}$ \\
\hline$\Delta$ Weight & $-21.7 \pm 13.0$ & $<0.0001$ & $-22.2 \pm 11.9$ & $<0.0001$ & $-21.4 \pm 15.6$ & $<0.0001$ & $-18.6 \pm 10.6$ & $<0.0001$ \\
\hline$\triangle \mathrm{BMI}$ & $-8.1 \pm 4.5$ & $<0.0001$ & $-8.0 \pm 4.1$ & $<0.0001$ & $-8.2 \pm 5.4$ & $<0.0001$ & $-7.9 \pm 4.1$ & $<0.0001$ \\
\hline$\Delta W C$ & $-15.4 \pm 10.1$ & $<0.0001$ & $-16.5 \pm 9.5$ & $<0.0001$ & $-13.6 \pm 11.8$ & $<0.0001$ & $-13.2 \pm 8.3$ & $<0.0001$ \\
\hline$\triangle F P G$ & $-15.6 \pm 25.8$ & $<0.0001$ & $-8.7 \pm 15.8$ & $<0.0001$ & $-13.7 \pm 17.5$ & $<0.0001$ & $-45.5 \pm 45.5$ & $<0.0001$ \\
\hline$\triangle 1 \mathrm{hPG}$ & $-36.7 \pm 48.8$ & $<0.0001$ & $-23.4 \pm 45.8$ & $<0.0001$ & $-48.4 \pm 47.2$ & $<0.0001$ & $-62.5 \pm 46.5$ & $<0.0001$ \\
\hline$\triangle 2 \mathrm{hPG}$ & $-34.7 \pm 53.9$ & $<0.0001^{*}$ & $-6.2 \pm 34.2$ & $0.1018^{*}$ & $-46.5 \pm 40.4$ & $<0.0001^{*}$ & $-113.5 \pm 53.7$ & $<0.0001^{*}$ \\
\hline$\Delta$ Insulin & $-8.6 \pm 9.7$ & $<0.0001$ & $-9.5 \pm 11.2$ & $<0.0001$ & $-6.9 \pm 7.2$ & $<0.0001$ & $-9.1 \pm 8.2$ & $<0.0001$ \\
\hline$\triangle \mathrm{HOMA}-\mathrm{IR}$ & $-2.8 \pm 3.3$ & $<0.0001$ & $-2.6 \pm 3.2$ & $<0.0001$ & $-2.3 \pm 2.4$ & $<0.0001$ & $-4.8 \pm 4.2$ & $<0.0001$ \\
\hline$\triangle \mathrm{HbAlc}$ & $-0.6 \pm 1.0$ & $<0.0001$ & $-0.3 \pm 0.6$ & $<0.0001$ & $-0.5 \pm 0.7$ & $<0.0001$ & $-1.7 \pm 1.7$ & $<0.0001$ \\
\hline$\Delta$ Total chol & $1.7 \pm 30.7$ & $0.4949^{*}$ & $0.0 \pm 29.8$ & $0.9971^{*}$ & $-0.2 \pm 31.7$ & $0.9711^{*}$ & $10.2 \pm 30.1$ & $0.1458^{*}$ \\
\hline$\triangle \mathrm{HDL}$-chol & $6.3 \pm 9.8$ & $<0.0001^{*}$ & $6.9 \pm 9.7$ & $<0.0001^{*}$ & $5.4 \pm 9.7$ & $0.0006^{*}$ & $5.2 \pm 9.9$ & $0.0386^{*}$ \\
\hline$\Delta$ Triglycerides & $-41.2 \pm 68.9$ & $<0.0001$ & $-34.6 \pm 59.6$ & $<0.0001$ & $-35.2 \pm 54.5$ & $<0.0001$ & $-76.5 \pm 104.9$ & 0.0019 \\
\hline$\triangle$ Systolic BP & $-5.2 \pm 14.7$ & 0.0001 & $-6.1 \pm 15.5$ & 0.0007 & $-1.4 \pm 11.4$ & 0.5372 & $-11.6 \pm 17.4$ & 0.0212 \\
\hline$\triangle$ Diastolic BP & $-3.2 \pm 11.2$ & 0.0014 & $-3.8 \pm 10.8$ & 0.0052 & $0.0 \pm 10.6$ & 0.9073 & $-8.8 \pm 12.6$ & 0.0114 \\
\hline$\Delta$ Creatinine & $0.0 \pm 0.1$ & 0.2882 & $0.0 \pm 0.1$ & 0.9522 & $0.0 \pm 0.1$ & 0.2774 & $0.0 \pm 0.1$ & 1.0000 \\
\hline$\Delta$ Leptin & $-17.7 \pm 12.8$ & $<0.0001^{*}$ & $-17.1 \pm 9.4$ & $<0.0001^{*}$ & $-21.1 \pm 16.4$ & $<0.0001^{*}$ & $-6.3 \pm 4.8$ & $0.0781^{*}$ \\
\hline$\Delta$ Ferritin & $-16.2 \pm 76.6$ & 0.0164 & $-18 \pm 44.7$ & 0.0095 & $-7.4 \pm 43.3$ & 0.5239 & $-31.1 \pm 190.6$ & 1.0000 \\
\hline$\Delta$ Transferrin & $-0.0 \pm 0.4$ & 0.3279 & $-0.0 \pm 0.4$ & 0.7014 & $-0.0 \pm 0.5$ & 0.5159 & $-0.1 \pm 0.4$ & 0.4132 \\
\hline$\Delta$ Iron & $1.8 \pm 28.7$ & 0.4731 & $2.2 \pm 30.0$ & 0.5303 & $3.6 \pm 27.2$ & 0.3900 & $-3.7 \pm 27.8$ & 0.5529 \\
\hline$\triangle$ Albumin & $-0.1 \pm 3.8$ & 0.8788 & $0.3 \pm 3.8$ & 0.4367 & $-0.5 \pm 4.2$ & 0.4401 & $-0.6 \pm 2.9$ & 0.3637 \\
\hline$\Delta$ Calcium & $0.0 \pm 0.1$ & $0.0055^{*}$ & $0.0 \pm 0.1$ & $0.0099^{*}$ & $0.0 \pm 0.1$ & $0.1731^{*}$ & $0.0 \pm 0.1$ & $0.8552^{*}$ \\
\hline$\triangle$ Sodium & $0.8 \pm 3.0$ & $0.0014^{*}$ & $0.5 \pm 3.2$ & $0.1291^{*}$ & $1.1 \pm 2.4$ & $0.0039^{*}$ & $1.5 \pm 2.9$ & $0.0321^{*}$ \\
\hline$\triangle$ Supine PRA & $-0.9 \pm 4.5$ & 0.0035 & $-0.4 \pm 3.8$ & 0.0067 & $-1.1 \pm 4.7$ & 0.2947 & $-3.1 \pm 7.0$ & 0.5195 \\
\hline$\triangle$ Upright PRA & $-1.0 \pm 7.9$ & 0.1821 & $0.0 \pm 7.2$ & 0.5256 & $-1.5 \pm 5.5$ & 0.2745 & $-5.0 \pm 14.5$ & 0.5186 \\
\hline$\triangle$ Supine ALD & $-0.6 \pm 4.3$ & 0.0005 & $-0.7 \pm 5.3$ & 0.0016 & $-0.4 \pm 2.0$ & 0.2624 & $-1.0 \pm 2.8$ & 0.2439 \\
\hline$\triangle$ Upright ALD & $-0.4 \pm 8.7$ & 0.0439 & $0.0 \pm 10.3$ & 0.1674 & $-0.1 \pm 4.5$ & 0.5056 & $-3.1 \pm 8.1$ & 0.1099 \\
\hline$\triangle$ Upright A/PRA & $-0.5 \pm 8.7$ & 0.8917 & $-0.1 \pm 9.3$ & 0.8980 & $-0.5 \pm 7.0$ & 0.2851 & $-2.9 \pm 9.9$ & 0.3804 \\
\hline$\Delta$ Urinary cortisol & $-15.9 \pm 56.3$ & $0.0170^{*}$ & $-21.5 \pm 49.9$ & $0.0060^{*}$ & $-9.7 \pm 68.8$ & $0.5379^{*}$ & $-3.1 \pm 58.8$ & $0.8714^{*}$ \\
\hline$\Delta$ Uric acid & $-0.4 \pm 1.1$ & $<0.0001^{*}$ & $-0.5 \pm 1.1$ & $<0.0001^{*}$ & $-0.4 \pm 1.1$ & $0.0138^{*}$ & $-0.2 \pm 1.5$ & $0.5167^{*}$ \\
\hline$\Delta$ Hypoglycemic $^{\mathrm{a}}$ & $0.0 \pm 1.2$ & 1.0000 & $0.0 \pm 0.0$ & N.E & $0.1 \pm 0.5$ & 1.0000 & $-0.2 \pm 3.4$ & 1.0000 \\
\hline
\end{tabular}

- indicates decrease

NGT normal glucose tolerance, IGT impaired glucose tolerance, T2D type 2 diabetes, $B M I$ body mass index, WC waist circumference, $F P G$ fasting plasma glucose, 1 hPG 1-hour plasma glucose, 2 PPG 2-hour plasma glucose, $B P$ blood pressure, $P R A$ plasma renin activity, $A L D$ aldosterone, $A L D / P R A$ Upright A/PRA, N.E not estimable $\wedge$ Non-parametric Wilcoxon signed rank test for paired data

*Paired T test

a Pills per day

about $90 \%$ down-regulation of $\mathrm{miR}-122$ and a reduction of miR-342-3p, miR-320, miR-139-5p and miR-146a might regulate metabolic processes such as the citric acid cycle (TCA) cycle, glucose transport, pentose phosphate pathway, fatty-acid synthesis, mitochondrial oxidation, gluconeogenesis, and glycolysis. Obesity is recognized as a major cause of hypertension, and the combination of obesity and hypertension is recognized as a pre-eminent cause of $\mathrm{CV}$ risk. An additional important factor in the $\mathrm{CV}$ risk associated with obesity and hypertension is the role played by obesity in the development of T2D. Efforts aimed at diminishing the incidence and impact of diabetes, therefore, including both lifestyle changes and the appropriate use of antihypertensive and anti-obesity therapies, are an essential part of the overall therapeutic plan. The recommendation to use RAAS inhibitors after coronary artery bypass grafting (CABG) in order to reduce MACE (major adverse cardiovascular events) [50] are consistent with our observation about the reliable predictive power of Renin after bariatric surgery, among a wide variety of different procedures. Finally, our results point to possible clinical implications. Selection of patients for bariatric surgery is still an open question. Determination of metabolically healthy obese subjects, 
Table 6 Changes induced by weight loss according to hypertension

\begin{tabular}{|c|c|c|c|c|}
\hline & NT & $p^{\wedge}$ & HT & $p^{\wedge}$ \\
\hline$\Delta$ Weight & $-22.0 \pm 12.2$ & $<0.0001$ & $-20.9 \pm 14.6$ & $<0.0001$ \\
\hline$\triangle \mathrm{BMl}$ & $-8.2 \pm 4.3$ & $<0.0001$ & $-7.9 \pm 5.0$ & $<0.0001$ \\
\hline$\Delta$ Waist circumference & $-15.4 \pm 10.0$ & $<0.0001$ & $-15.3 \pm 10.3$ & $<0.0001$ \\
\hline$\triangle F P G$ & $-11.5 \pm 18.2$ & $<0.0001$ & $-24.1 \pm 35.7$ & $<0.0001$ \\
\hline$\triangle 1 \mathrm{hPG}$ & $-34.3 \pm 45.3$ & $<0.0001$ & $-41.5 \pm 55.6$ & $<0.0001$ \\
\hline$\triangle 2 \mathrm{hPG}$ & $-28.1 \pm 45.9$ & $<0.0001^{*}$ & $-48.1 \pm 65.8$ & $<0.0001^{*}$ \\
\hline$\Delta$ Insulin & $-8.8 \pm 10.6$ & $<0.0001$ & $-8.2 \pm 7.6$ & $<0.0001$ \\
\hline$\triangle \mathrm{HOMA}-\mathrm{IR}$ & $-2.5 \pm 3.1$ & $<0.0001$ & $-3.3 \pm 3.5$ & $<0.0001$ \\
\hline$\triangle \mathrm{HbA} 1 \mathrm{c}$ & $-0.5 \pm 0.9$ & $<0.0001$ & $-0.9 \pm 1.1$ & $<0.0001$ \\
\hline$\Delta$ Total cholesterol & $0.5 \pm 29.5$ & $0.8489^{*}$ & $4.0 \pm 33.2$ & $0.3935^{*}$ \\
\hline$\triangle \mathrm{HDL}$-cholesterol & $6.2 \pm 9.7$ & $<0.0001^{*}$ & $6.7 \pm 10.1$ & $<0.0001^{*}$ \\
\hline$\Delta$ Triglycerides & $-38.8 \pm 65.0$ & $<0.0001$ & $-46.4 \pm 76.9$ & $<0.0001$ \\
\hline$\triangle$ Systolic BP & $-2.8 \pm 11.4$ & 0.0156 & $-9.0 \pm 18.4$ & 0.0003 \\
\hline$\triangle$ Diastolic BP & $-1.4 \pm 9.7$ & 0.0948 & $-5.8 \pm 12.2$ & 0.0004 \\
\hline$\Delta$ Creatinine & $0.0 \pm 0.1$ & 0.7750 & $0.0 \pm 0.1$ & 0.1481 \\
\hline$\Delta$ Leptin & $-19.1 \pm 12.9$ & $<0.0001$ & $-15.5 \pm 12.6$ & 0.0002 \\
\hline$\Delta$ Ferritin & $-15.9 \pm 41.8$ & 0.0042 & $-16.8 \pm 125.5$ & 0.8130 \\
\hline$\Delta$ Transferrin & $0.0 \pm 0.4$ & 0.5693 & $0.1 \pm 0.4$ & 0.3586 \\
\hline$\Delta$ Iron & $1.7 \pm 28.8$ & 0.5846 & $1.9 \pm 28.9$ & 0.6453 \\
\hline$\triangle$ Albumin & $0.2 \pm 4.2$ & 0.6064 & $-0.2 \pm 3.7$ & 0.6609 \\
\hline$\Delta$ Calcium & $0.04 \pm 0.12$ & $0.0037^{*}$ & $0.02 \pm 0.11$ & $0.1442^{*}$ \\
\hline$\triangle$ Sodium & $0.41 \pm 0.30$ & $0.2717^{*}$ & $1.4 \pm 0.36$ & $<0.0001^{*}$ \\
\hline$\triangle$ Supine PRA & $0.1 \pm 3.0$ & 0.3616 & $-2.7 \pm 6.0$ & 0.0005 \\
\hline$\triangle$ Upright PRA & $0.4 \pm 6.0$ & 0.8130 & $-3.6 \pm 10.0$ & 0.0605 \\
\hline$\triangle$ Supine ALD & $-0.2 \pm 4.8$ & 0.0529 & $-1.4 \pm 3.2$ & 0.0015 \\
\hline$\triangle$ Upright ALD & $0.3 \pm 10.0$ & 0.2545 & $-1.6 \pm 5.5$ & 0.0796 \\
\hline$\triangle$ Upright ALD/PRA & $-0.5 \pm 8.2$ & 0.7108 & $-0.7 \pm 9.6$ & 0.5834 \\
\hline$\Delta$ Urinary free cortisol & $-13.4 \pm 56.5$ & $0.1085^{*}$ & $-20.4 \pm 56.7$ & $0.0731^{*}$ \\
\hline$\Delta$ Uric-acid & $-0.4 \pm 1.2$ & $0.0009^{*}$ & $-0.5 \pm 1.0$ & $0.0011^{*}$ \\
\hline$\Delta$ Hypoglycemic therapy $^{a}$ & $0.1 \pm 1.2$ & 0.7500 & $-0.1 \pm 1.2$ & 1.0000 \\
\hline
\end{tabular}

- indicates decrease

$N T$ normotensive, $H T$ hypertensive, BMI body mass index, FPG fasting plasma glucose, 1 hPG 1-hour plasma glucose, 2 PPG 2-hour plasma glucose, $B P$ blood pressure, PRA plasma renin activity, $A L D$ aldosterone

${ }^{\wedge}$ Non-parametric Wilcoxon signed rank test for paired data

*Paired T test

a Pills per day

for whom surgery is probably not indicated, seems possible only through genetic studies [51]. The response of individual subjects to surgery depends on the aim of surgery; for instance, prevention of mortality is more effective above a given age, while prevention of diabetes is valid for all ages [52]. Response to surgery also depends on age of patients, on initial BMI, and on the type of surgery [53, 54]. Remission of diabetes also depends on the duration of diabetes [55]. The predictive model of persistent hypertension in spite of weight loss is promising, but it should be confirmed for other surgeries, for instance malabsorptive surgeries (biliopancreatic diversion), mixed surgeries (gastric bypass), or restrictive surgeries (sleeve gastrectomy), all more effective than LAGB [54]. In the meanwhile, patients with persistent hypertension should receive close supervision and more intensive treatment to lower blood pressure, even because left ventricular hypertrophy does not regress in hypertensive patients [56]. 
Table 7 Logistic models for the probability of hypertension 12 months after LAGB

\begin{tabular}{|c|c|c|c|c|c|c|}
\hline \multirow[t]{2}{*}{ Variables } & \multicolumn{2}{|l|}{ Model 1} & \multicolumn{2}{|l|}{ Model 2} & \multicolumn{2}{|l|}{ Model 3} \\
\hline & OR $(95 \% \mathrm{Cl})$ & $p$ & OR $(95 \% \mathrm{Cl})$ & $p$ & OR $(95 \% \mathrm{Cl})$ & $p$ \\
\hline Supine PRA (ng/ml/h) & $1.63(1.19-2.24)$ & 0.0023 & $1.47(1.10-1.96)$ & 0.0085 & $1.52(1.14-2.03)$ & 0.0049 \\
\hline \multicolumn{7}{|c|}{ Glucose tolerance groups } \\
\hline NGT & 1.00 (Reference) & & - & - & - & - \\
\hline IGT & $3.60(1.22-10.60)$ & 0.0319 & - & - & - & - \\
\hline $\mathrm{T} 2 \mathrm{D}$ & $4.85(1.15-20.56)$ & 0.0201 & - & - & - & - \\
\hline FPG & - & - & $1.02(1.00-1.03)$ & 0.0453 & - & - \\
\hline \multirow[t]{2}{*}{$2 \mathrm{hPG}$} & - & - & - & - & $1.01(1.00-1.02)$ & 0.0470 \\
\hline & $A \cup C=0.784$ & & $A \cup C=0.782$ & & $A \cup C=0.770$ & \\
\hline
\end{tabular}

Model 1 is based on supine PRA and glucose tolerance groups. Model 2 is based on supine PRA and FPG. Model 3 is based on supine PRA and 2hPG OR odds ratio, $C l$ confidence interval, PRA plasma renin activity, NGT normal glucose tolerance, IGT impaired glucose tolerance, T2D Type 2 diabetes, FPG fasting plasma glucose, $2 h P G$ 2-hour plasma glucose, $A U C$ area under the curve
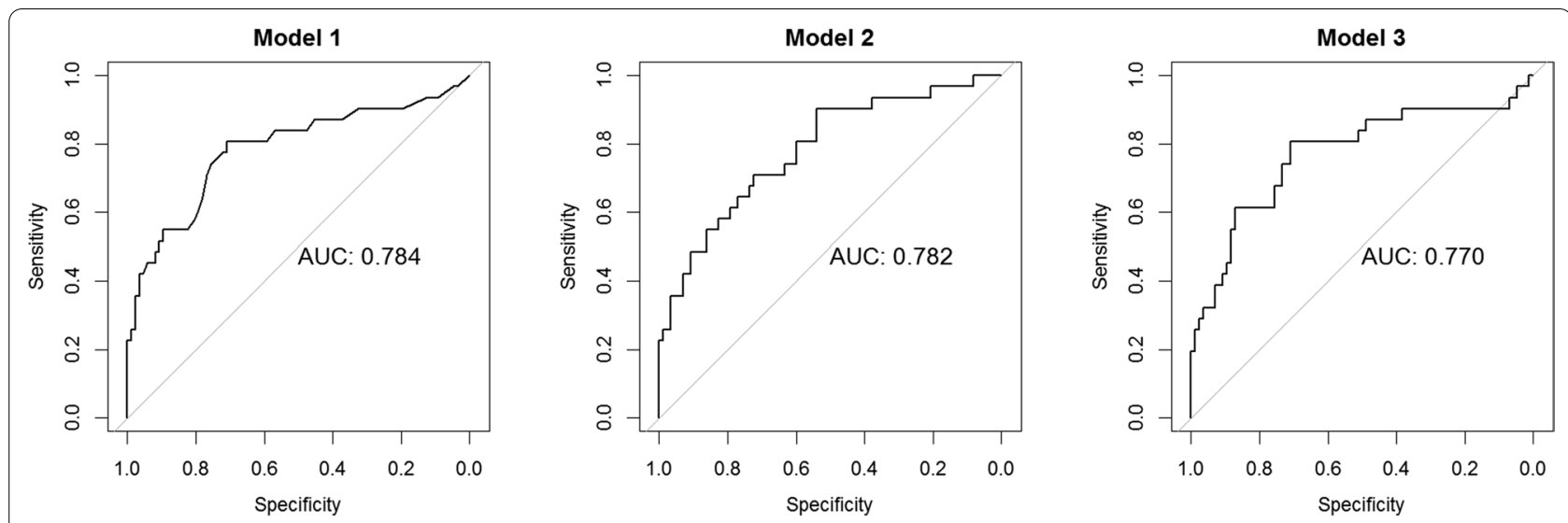

Fig. 2 Receiver operating characteristic (ROC) curves and AUC (area under the ROC curve) of logistic regression models for the probability of hypertension at 12 months after laparoscopic adjustable gastric banding (LAGB)

\section{Conclusions}

In obese subjects supine PRA is higher in hypertensive than in normotensive subjects, with a trend for progressively higher with normal glucose tolerance, impaired glucose tolerance and diabetes. Supine PRA correlates with HOMA-IR, and interestingly that both PRA and HOMA-IR correlate with ferritin. Decrease of supine PRA at 1 year after BS only occurs in hypertensive subjects, as previously reported, with no difference among groups of glucose tolerance. Decrease of supine PRA correlated with decrease of HOMA-IR and with decrease of ferritin, suggesting that changes of ferritin are mechanistically linked to change of HOMAIR and of supine PRA. Similar, albeit less significant, was the behavior of upright PRA, and of both supine and upright aldosterone. Higher supine PRA and worse glucose tolerance (and FPG and 2hPG) predict persistence of hypertension after bariatric surgery in spite of weight loss. 


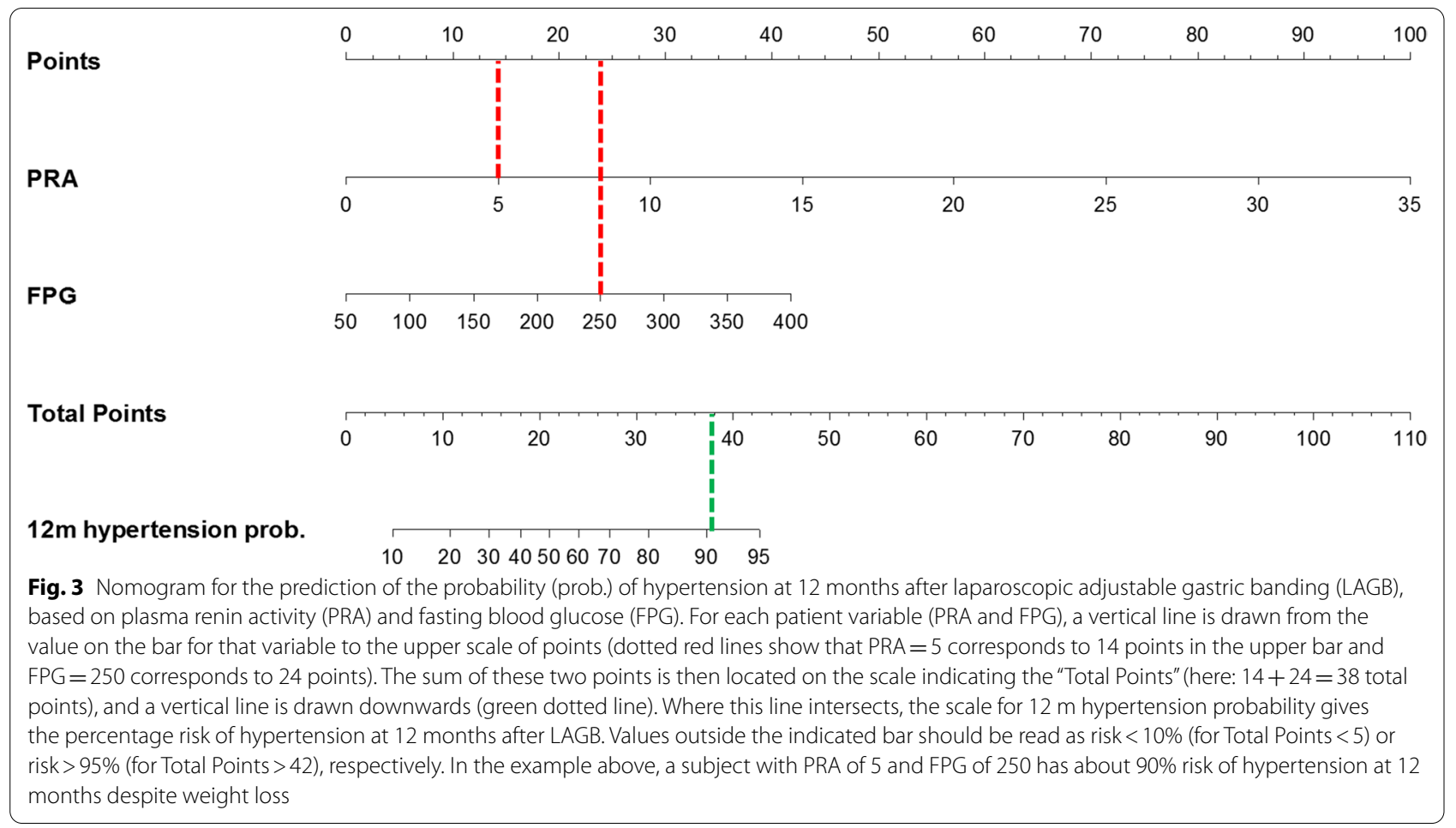

\section{Abbreviations}

PRA: Plasma renin activity (supine and upright); ALD: Aldosterone (supine and upright); HbA1c: Haemoglobin A1 c; FPG: Fasting plasma glucose; 1hPG: 1h plasma glucose after OGTT; 2hPG: 2h plasma glucose after OGTT; HOMA-IR: Homeostasis model assessment for insulinresistance; OGTT: Oral glucose tolerance test; BMI: Body mass index; GT: Glucose tolerance (NGT=normal; $\mathrm{IGT}=$ impaired; $\mathrm{T} 2 \mathrm{D}=$ type 2 diabetes)

\section{Acknowledgments}

The authors would like to thank Fondazione Romeo and Enrica Invernizzi, Milan (Italy), for the support.

\section{Authors' contributions}

Design of the study: LLS, FF, AEP; search of data: LLS, ET and AEP; analysis of data: ET; writing - original draft preparation: LLS and AEP; review and editing: LLS, ET, EV, FF, and AEP; final version: LLS, FF, and AEP. All authors read and approved the final manuscript.

\section{Funding}

This research was funded by Università degli Studi di Milano, Milan (Italy), by IRCCS MultiMedica, Milan (Italy), and Ministry of Health Ricerca Corrente, Italy to IRCCS MultiMedica, Milan (Italy).

\section{Availability of data and materials}

Data are available on request.

\section{Declarations}

Ethics approval and consent to partricipate

The protocol of bariatric surgery has been approved by the Local Ethics Committee in 1995, in 2002, and in 2006.

\section{Consent for publication}

Not applicable.

\section{Competing interests}

The authors declare no conflict of interest.

\section{Author details}

${ }^{1}$ Lab. of Cardiovascular and Dysmetabolic Disease, IRCCS MultiMedica, 20138 Milan, Italy. ${ }^{2}$ Value-based Healthcare Unit, IRCCS MultiMedica, Milan, Italy. ${ }^{3}$ Postgraduate Program on Physical Education, Universidade Católica de Brasília, Taguatinga, DF 71966-700, Brazil. ${ }^{4}$ Dipartimento di Scienze della Salute, Università degli Studi di Milano, 20142 Milan, Italy. ${ }^{5}$ Unità di Endocrinologia, Ospedale San Paolo, ASST Santi Paolo e Carlo, Milan, Italy.

Received: 22 May 2021 Accepted: 2 June 2021

Published online: 09 June 2021

\section{References}

1. Fuchs FD, Whelton PK. High blood pressure and cardiovascular disease. Hypertension. 2020;75:285-92.

2. Lewington S, Clarke R, Qizilbash N, Peto R, Collins R, Prospective Studies Collaboration. Prospective studies. Age-specific relevance of usual blood pressure to vascular mortality: a meta-analysis of individual data for one million adults in 61 prospective studies. Lancet. 2002;360:1903-13.

3. Kannel WB. The Framingham study: ITS 50-year legacy and future promise. J Atheroscler Thromb. 2000;6:60-6.

4. Rowe JW, Young JB, Minaker KL, Stevens AL, Pallotta J, Landsberg L. Effect of insulin and glucose infusions on sympathetic nervous system activity in normal man. Diabetes. 1981;30:219-25.

5. Perego L, Pizzocri P, Corradi D, Maisano F, Paganelli M, Fiorina P, Barbieri M, Morabito A, Paolisso G, Folli F, Pontiroli AE. Circulating leptin correlates with left ventricular mass in morbid (grade III) obesity before and after weight loss induced by bariatric surgery: a potential role for leptin in mediating human left ventricular hypertrophy. J Clin Endocrinol Metab. 2005:90:4087-93. 
6. Hall JE, do Carmo JM, da Silva AA, Wang Z, Hall ME. Obesity, kidney dysfunction and hypertension: mechanistic links. Nat Rev Nephrol. 2019:15:367-85.

7. Fiorentino TV, Prioletta A, Zuo P, Folli F. Hyperglycemia-induced oxidative stress and its role in diabetes mellitus related cardiovascular diseases. Curr Pharm Des. 2013;19:5695-703.

8. Ferrannini E, Buzzigoli G, Bonadonna R, Giorico MA, Oleggini M, Graziadei L, Pedrinelli R, Brandi L, Bevilacqua S. Insulin resistance in essential hypertension. N Engl J Med. 1987;317:350-7.

9. Velloso LA, Folli F, Sun XJ, White MF, Saad MJ, Kahn CR. Cross-talk between the insulin and angiotensin signaling systems. Proc Natl Acad Sci USA. 1996;93:12490-5.

10. Magkos F, Hjorth MF, Astrup A. Diet and exercise in the prevention and treatment of type 2 diabetes mellitus. Nat Rev Endocrinol. 2020;16(10):545-55.

11. Arterburn DE, Telem DA, Kushner RF, Courcoulas AP. Benefits and risks of bariatric surgery in adults: a review. JAMA. 2020;324:879-87.

12. La Sala L, Pontiroli AE. Prevention of diabetes and cardiovascular disease in obesity. Int J Mol Sci. 2020;21(21):8178.

13. Heffron SP, Parham JS, Pendse J, Alemán JO. Treatment of obesity in mitigating metabolic risk. Circ Res. 2020;126:1646-65.

14. Pontiroli AE, Morabito A. Long-term prevention of mortality in morbid obesity through bariatric surgery. a systematic review and meta-analysis of trials performed with gastric banding and gastric bypass. Ann Surg. 2011;253:484-7.

15. Raaijmakers LC, Pouwels S, Thomassen SE, Nienhuijs SW. Quality of life and bariatric surgery: a systematic review of short- and longterm results and comparison with community norms. Eur J Clin Nutr. 2017;71:441-9

16. Picot J, Jones J, Colquitt JL, Gospodarevskaya E, Loveman E, Baxter L, Clegg AJ. The clinical effectiveness and cost-effectiveness of bariatric (weight loss) surgery for obesity: a systematic review and economic evaluation. Health Technol Assess. 2009;13:1-190.

17. Keating $C L$, Dixon JB, Moodie ML, Peeters A, Bulfone L, Maglianno DJ, O'Brien PE. Cost-effectiveness of surgically induced weight loss for the management of type 2 diabetes: modeled lifetime analysis. Diabetes Care. 2009;32:567-74

18. Pontiroli $A E$, Frigè $F$, Paganelli $M$, Folli $F$. In morbid obesity, metabolic abnormalities and adhesion molecules correlate with visceral fat, not with subcutaneous fat: effect of weight loss through surgery. Obes Surg. 2009;19:745-50

19. Heneghan HM, Meron-Eldar S, Brethauer SA, Schauer PR, Young JB. Effect of bariatric surgery on cardiovascular risk profile. Am J Cardiol. 2011;108:1499-507.

20. Dall'Asta C, Vedani P, Manunta P, Pizzocri P, Marchi M, Paganelli M, Folli F, Pontiroli AE. Effect of weight loss through laparoscopic gastric banding on blood pressure, plasma renin activity and aldosterone levels in morbid obesity. Nutr Metab Cardiovasc Dis. 2009;19:110-4.

21. Joseph JJ, Echouffo Tcheugui JB, Effoe VS, Hsueh WA, Allison MA, Golden SH. Renin-angiotensin-aldosterone system, glucose metabolism and incident type 2 diabetes mellitus: MESA. J Am Heart Assoc. 2018;7:e009890

22. Scheen AJ. Renin-angiotensin system inhibition prevents type 2 diabetes mellitus. Part 1. A meta-analysis of randomised clinical trials. Diabetes Metab. 2004;30:487-96.

23. Scheen AJ. Renin-angiotensin system inhibition prevents type 2 diabetes mellitus. Part 2. Overview of physiological and biochemical mechanisms. Diabetes Metab. 2004:30:498-505.

24. Pontiroli AE, Pizzocri P, Librenti MC, Vedani P, Marchi M, Cucchi E, Orena C, Paganelli M, Giacomelli M, Ferla G, Folli F. Laparoscopic adjustable gastric banding for the treatment of morbid (grade 3) obesity and its metabolic complications: a three year study. J Clin Endocrinol Metab. 2002;87:3555-61.

25. The Joint National Committee on Prevention, Detection, Evaluation and Treatment of High Blood Pressure. The sixth report of Joint National Committee on prevention, detection, evaluation and treatment of high blood pressure. Arch Intern Med. 1997;157:2413-46.

26. Matthews DR, Hosker JP, Rudenski AS, Naylor BA, Treacher DF, Turner RC. Homeostasis model assessment: insulin resistance and beta-cell function from fasting plasma glucose and insulin concentrations in man. Diabetologia. 1985;28:412-9.
27. Giacchetti G, Ronconi V, Lucarelli G, Boscaro M, Mantero F. Analysis of screening and confirmatory test in the diagnosis of primary aldosteronism: need for a standardized protocol. J Hypertens. 2006;24:737-45.

28. Pazzagli M, Kim JB, Messeri G, Kohen F, Bolelli GF, Tommasi A, Salerno $\mathrm{R}$, Serio M. Luminescent immunoassay (LIA) ofcortisol-2. Development and validation of the immunoassay monitored by chemiluminescence. J Steroid Biochem. 1981;14:1181-7.

29. Krasowski MD, Drees D, Morris CS, Maakestad J, Blau JL, Ekins S. Crossreactivity of steroid hormone immunoassays: clinical significance and two-dimensional molecular similarity prediction. BMC Clin Pathol. 2014;14:33.

30. Pontiroli AE, Folli F, Paganelli M, Micheletto G, Pizzocri P, Vedani P, Luisi F, Perego L, Morabito A, Bressani Doldi S. Laparoscopic gastric banding prevents type 2 diabetes and arterial hypertension and induces their remission in morbid obesity: a 4 year case-controlled study. Diabetes Care. 2005;28:2703-9.

31. Gastaldelli A, Perego L, Paganelli M, Sesti G, Hribal M, Chavez AO, Defronzo RA, Pontiroli A, Folli F. Elevated concentrations of liver enzymes and ferritin identify a new phenotype of insulin resistance: effect of weight loss after gastric banding. Obes Surg. 2009;19:80-6.

32. Zhang C, Zhang J, Liu Z, Zhou Z. More than an anti-diabetic bariatric surgery, metabolic surgery alleviates systemic and local inflammation in obesity. Obes Surg. 2018;28:3658-68.

33. Kernan KF, Carcillo JA. Hyperferritinemia and inflammation. Int Immunol. 2017;29:401-9.

34. Muraca E, Ciardullo S, Perra S, Zerbini F, Oltolini A, Cannistraci R, Bianconi E, Villa M, Pizzi M, Pizzi P, Manzoni G, Lattuada G, Perseghin G. Hypercortisolism and altered glucose homeostasis in obese patients in the prebariatric surgery assessment. Diabetes Metab Res Rev. 2020 Aug;1:e3389.

35. Ceccato F, Lizzul L, Barbot M, Scaroni C. Pituitary-adrenal axis and peripheral cortisol metabolism in obese patients. Endocrine. 2020;69:386-92.

36. Werdermann M, Berger I, Scriba LD, Santambrogio A, Schlinkert P, Brendel $H$, Morawietz H, Schedl A, Peitzsch M, King AJF, Andoniadou CL, Bornstein SR, Steenblock C. Insulin and obesity transform hypothalamic-pituitaryadrenal axis stemness and function in a hyperactive state. Mol Metab. 2021:43:101112.

37. Folli F, Kahn CR, Hansen H, Bouchie JL, Feener EP. Angiotensin II inhibits insulin signaling in aortic smooth muscle cells at multiple levels. A potential role for serine phosphorylation in insulin/angiotensin II crosstalk. J Clin Invest. 1997;100:2158-69.

38. Federici M, Hribal ML, Menghini R, Kanno H, Marchetti , Porzio O, Sunnarborg SW, Rizza S, Serino M, Cunsolo V, Lauro D, Mauriello A, Smookler DS, Sbraccia P, Sesti G, Lee DC, Khokha R, Accili D, Lauro R. Timp3 deficiency in insulin receptor-haploinsufficient mice promotes diabetes and vascular inflammation via increased TNF-alpha. J Clin Invest. 2005;115:3494-505.

39. Monroy A, Kamath S, Chavez AO, Centonze VE, Veerasamy M, Barrentine A, Wewer JJ, Coletta DK, Jenkinson C, Jhingan RM, Smokler D, Reyna S, Musi N, Khokka R, Federici M, Tripathy D, DeFronzo RA, Folli F. Impaired regulation of the TNF-alpha converting enzyme/tissue inhibitor of metalloproteinase 3 proteolytic system in skeletal muscle of obese type 2 diabetic patients: a new mechanism of insulin resistance in humans. Diabetologia. 2009;52:2169-81.

40. Hotamisligil GS. Inflammation, metaflammation and immunometabolic disorders. Nature. 2017;542:177-85.

41. Fiorentino TV, Monroy A, Kamath S, Sotero R, Cas MD, Daniele G, Chavez AO, Abdul-Ghani M, Hribal ML, Sesti G, Tripathy D, DeFronzo RA, Folli F. Pioglitazone corrects dysregulation of skeletal muscle mitochondrial proteins involved in ATP synthesis in type 2 diabetes. Metabolism. 2021;114:154416.

42. Takahashi N, Li F, Hua K, Deng J, Wang CH, Bowers RR, Bartness TJ, Kim HS, Harp JB. Increased energy expenditure, dietary fat wasting, and resistance to diet-induced obesity in mice lacking renin. Cell Metab. 2007;6:506-12.

43. Gratze P, Boschmann M, Dechend R, Qadri F, Malchow J, Graeske S, Engeli S, Janke J, Springer J, Contrepas A, Plehm R, Klaus S, Nguyen G, Luft FC, Muller DN. Energy metabolism in human renin-gene transgenic rats: does renin contribute to obesity? Hypertension. 2009;53:516-23.

44. La Sala L, Crestani M, Garavelli S, de Candia P, Pontiroli AE. Does microRNA perturbation control the mechanisms linking obesity and diabetes? Implications for cardiovascular risk. Int J Mol Sci. 2020;22(1):143. 
45. Yu Y, Du H, Wei S, Feng L, Li J, Yao F, Zhang M, Hatch GM, Chen L. Adipocyte-derived exosomal MiR-27a Induces insulin resistance in skeletal muscle through repression of PPARY. Theranostics. 2018;8:2171-88.

46. Madsbad S, Dirksen C, Holst JJ. Mechanisms of changes in glucose metabolism and bodyweight after bariatric surgery. Lancet Diabetes Endocrinol. 2014;2:152-64.

47. Hubal MJ, Nadler EP, Ferrante SC, Barberio MD, Suh JH, Wang J, Dohm GL, Pories WJ, Mietus-Snyder M, Freishtat RJ. Circulating adipocyte-derived exosomal MicroRNAs associated with decreased insulin resistance after gastric bypass. Obesity. 2017;25:102-10.

48. Kuryłowicz A, Wicik Z, Owczarz M, Jonas MI, Kotlarek M, Świerniak M, Lisik W, Jonas M, Noszczyk B, Puzianowska-Kuźnicka M. NGS reveals molecular pathways affected by obesity and weight loss-related changes in miRNA levels in adipose tissue. Int J Mol Sci. 2017;19(1):66.

49. Wu Q, Li JV, Seyfried F, le Roux CW, Ashrafian H, Athanasiou T, Fenske W, Darzi A, Nicholson JK, Holmes E, Gooderham NJ. Metabolic phenotypemicroRNA data fusion analysis of the systemic consequences of Roux-enY gastric bypass surgery. Int J Obes. 2015;39:1126-34.

50. Martinsson A, Nielsen SJ, Björklund E, Pivodic A, Malm CJ, Hansson EC, Jeppsson A. Renin-angiotensin system inhibition and outcome after coronary artery bypass grafting: a population-based study from the SWEDEHEART registry. Int J Cardiol. 2021;331:40-5.

51. Huang LO, Rauch A, Mazzaferro E, Preuss M, Carobbio S, Bayrak CS, Chami N, Wang Z, Schick UM, Yang N, Itan Y, Vidal-Puig A, den Hoed M, Mandrup S, Kilpeläinen TO, Loos RJF. Genome-wide discovery of genetic loci that uncouple excess adiposity from its comorbidities. Nat Metab. 2021;3:228-43.

52. Pontiroli AE, Ceriani V, Tagliabue E. Compared with controls, bariatric surgery prevents long-term mortality in persons with obesity only above median age of cohorts: a systematic review and meta-analysis. Obes Surg. 2020;30:2487-96.

53. Nickel F, de la Garza JR, Werthmann FS, Benner L, Tapking C, Karadza E, Wekerle AL, Billeter AT, Kenngott HG, Fischer L, Müller-Stich BP. Predictors of risk and success of obesity surgery. Obes Facts. 2019;12:427-39.

54. Buchwald H, Avidor $Y$, Braunwald E, Jensen MD, Pories W, Fahrbach K, Schoelles K. Bariatric surgery: a systematic review and meta-analysis. JAMA. 2004;292:1724-37.

55. Aung L, Lee WJ, Chen SC, Ser KH, Wu CC, Chong K, Lee YC, Chen JC. Bariatric surgery for patients with early-onset vs late-onset type 2 diabetes. JAMA Surg. 2016;151:798-805.

56. Pontiroli AE, Pizzocri P, Saibene A, Girola A, Koprivec D, Fragasso G. Left ventricular hypertrophy and QT interval in obesity and in hypertension: effects of weight loss and of normalisation of blood pressure. Int J Obes Relat Metab Disord. 2004;28:1118-23.

\section{Publisher's Note}

Springer Nature remains neutral with regard to jurisdictional claims in published maps and institutional affiliations.
Ready to submit your research? Choose BMC and benefit from:

- fast, convenient online submission

- thorough peer review by experienced researchers in your field

- rapid publication on acceptance

- support for research data, including large and complex data types

- gold Open Access which fosters wider collaboration and increased citations

- maximum visibility for your research: over $100 \mathrm{M}$ website views per year

At BMC, research is always in progress.

Learn more biomedcentral.com/submissions 